\title{
Funktionelle Morphologie des Geruchsorgans und Histologie der Kopfanhänge der Nasenmuräne Rbinomuraena ambonensis (Teleostei, Anguilliformes)
}

\author{
A. Holl, E. Schulte und W. Meinel \\ 1. Zoologisches Institut der Justus-Liebig-Universität Gießen; \\ Gießen/Labn, Deutschland (BRD)
}

\begin{abstract}
Functional morphology of the olfactory organ and histology of the head appendages of the moray eel Rbinomuraena ambonensis (Teleostei, Anguilliformes). In three individuals of the moray eel Rbinomuraena ambonensis (BARBOUR), the functional morphology and histology of the olfactory organ as well as the histology of the different head appendages were investigated. The edges of the anterior nares are extremely extended, forming a funnellike wide opening through which the odour water passes. Even when the fish does not move, an effective ventilation of the olfactory chambers is maintained by a permanent water current towards the funnel opening induced by the peristaltic movements of the musculature of the gill chamber (principle of BerNoulur). The histological structure of the head appendages (funnels, rostrum and mandibulary barbels, which are all skin projections) is described. The epithelia of all these appendages bear sensory organs which are similar to taste buds. The two olfactory organs are of extremely large size. Each chamber contains about 100 olfactory lamellae which are arranged in two lines $\left(9^{\circ}\right.$-type; Holl 1965$)$. A cylindrical cavity extends between the inner edges of the olfactory lamellae through which the water runs to the posterior nare. Interlamellar microventilation is produced by numerous turbulences which are caused by the edges of the olfactory lamellae and by the kinocilia of the olfactory epithelium. The histological structure of the olfactory epithelium is similar to that of Anguilla anguilla. The different results demonstrate that Rhinomuraena ambonersis is probably a macrosmatic fish.
\end{abstract}

\section{EINLEITUNG}

Das innerhalb des Chordaten-Stammes erstmals bei niederen Fischen auftretende Geruchsorgan erreicht bei Knorpel- und Knochenfischen als wichtigstes chemisches Sinnesorgan eine bemerkenswert hohe Entwicklungsstufe. Während es bei den Knorpelfischen eine weitgehend uniforme morphologische und auch histologische Strukturierung aufweist, zeigt - soweit bekannt - sein Aufbau bei den Knochenfischen eine sehr verschiedenartige Differenzierung, zumindest bei einem Teil der zahlreichen Familien. Diese Differenzierung steht in Zusammenhang mit der Lebensweise der Arten. Nach Untersuchungen von TErchmanN (1954) lassen sich die Knochenfische des Süßwassers hinsichtlich der Ausbildung und Leistung des Geruchsorgans, wobei auch die Bedeutung des Auges berücksichtigt wird, in drei Gruppen einteilen. Vergleichende Un- 
tersuchungen über den makroskopischen und mikroskopischen Bau des Geruchsorgans bei marinen Arten liegen zwar vor (BUrne 1909, Holl 1964, 1969, Pferffer 1964, u. a.) es fehlt jedoch - mit Ausnahme der Bearbeitung von Aphanopus carbo (Holl \& Meinel 1969) - die Einordnung in das von TeichmanN (1954) aufgestellte System ebenso wie die Behandlung der funktionellen Morphologie.

Im Rahmen einer umfassenderen, vergleichenden Bearbeitung des Geruchsorgans litoraler Knochenfische hatten die Verfasser Gelegenheit, zwei lebende Exemplare der bis vor kurzem als Seltenheit anzusehenden Nasenmuräne, Rbinomuraena ambonensis (BARBOUR) zu untersuchen.

Die beiden bisher bekannten Arten der Gattung Rhinomuraena, $R$. ambonensis und $R$. quasita (Weber \& Beaufort 1916, Kobayashr 1962, Schultz et al. 1953, KLAusewitz 1969), bewohnen - soweit bekannt - die Sandzonen der Küstengebiete und die Spalten von Korallenstöcken im Indischen und Pazifischen Ozean, sind also typische Vertreter der Litoralfauna.

Von besonderem Interesse bei der Gattung Rhinomuraena ist das Geruchsorgan, das schon durch die extrem vergrößerten Ränder der vorderen Riechöffnungen in seiner äußeren Gestaltung auffällt. Aber nicht allein die großen trichterförmigen Auswüchse weisen darauf hin, daß bei der Gattung Rhinomuraena der Geruchssinn bei der Orientierung eine wichtige Rolle zu spielen scheint, sondern auch die außerordentlich hohe Zahl der Riechfalten, die als Riechfaltensystem den größten Teil des vor den - durchaus nicht kleinen - Augen gelegenen Kopfbereiches einnehmen. Der Name Rbinomuraena ist also sehr treffend gewählt, obwohl bisher keinerlei Einzelheiten über Größe, Bau und Bedeutung des Geruchsorgans von Rhinomuraena bekannt waren.

Ziel der vorliegenden Untersuchungen war es, die Morphologie und Histologie des Geruchsorgans von Rhinomuraena ambonensis $\mathrm{zu}$ bearbeiten, dabei aber auch Näheres über die funktionelle Morphologie der beiden Nasentrichter in Erfahrung zu bringen. Daneben erschien es angebracht, den histologischen Bau des Rostrum und der Barteln einer näheren Betrachtung zu unterziehen und der Frage nachzugehen, ob und inwieweit Sinnesorgane auf Rostrum und Barteln existieren.

\section{MA'TERIAL UND METHODIK}

Für die vorliegenden Untersuchungen standen drei Tiere der Gattung Rbinomuraena ambonensis (BARBOUR) zur Verfügung, von denen zwei Importe vom Aquariumhandel lebend bezogen wurden. Ein drittes Tier wurde uns freundlicherweise von Herrn Dr. KLAusewitz (Frankfurt/M.) überlassen.

Bei allen. Individuen dürfte es sich um annähernd ausgewachsene Tiere gehandelt haben, denn wesentlich größere Tiere wurden in den verschiedensten Seewasseraquarien (bei Zoogroßhändlern, sowie in mehreren Exotarien) nirgends angetroffen, wobei vorausgesetzt werden muß, daß verschiedene Fangorte vorliegen. Wahrscheinlich stammten die hier untersuchten Tiere aus dem Gebiet der Philippinen.

Die nach der Fixierung gemessene Länge der drei Tiere betrug: $82 \mathrm{~cm}, 89 \mathrm{~cm}$ und $96 \mathrm{~cm}$. Die beiden vom Zoohandel erworbenen Fische wurden fast zwei Monate in 
künstlichem Seewasser gehältert. Während dieser Zeit wurde weder eine Futteraufnahme beobachtet, noch wies die spätere Magenuntersuchung auf eine solche hin. Auch das künstliche Seewasser schien ihnen nicht zu behagen, denn mehrmals versuchten sie das Aquarium zu verlassen, und zwar mit dem Schwanz zuerst. Daher war es notwendig, einen Abstand von mindestens $15 \mathrm{~cm}$ zwischen Wasseroberfläche und Aquariumrand zu halten.

Für die Untersuchungen zur Frage der Duftwasserströmung beim ruhenden Fisch wurde eine Tuschelösung mit Hilfe einer Pipette in unmittelbare Nähe der Trichteröffnung gegeben. $\mathrm{Da}$ die Tiere dann in den meisten Fällen rückwärts schwammen, mußten sie einer leichten Narkose mit MS 222 unterzogen werden, die nur so weit ging, daß gerade die Fluchtreaktion (nach vor- oder rückwärts) ausgeschaltet war. Die Tuscheexperimente an der eröffneten Riechhöhle machten eine Vollnarkose der Tiere notwendig.

Für die histologische Bearbeitung wurden nur die beiden in Aquarium gehaltenen Fische herangezogen. Der Kopf des kleineren Tieres wurde in Bouin fixiert, der Kopf des größeren in $7 \%$ igem, neutralem Formalin. Die Nachbehandlung der präparierten Geruchsorgane und Kopfanhänge erfolgte in Isopropanol und die Einbettung über Isopropanol-Paraffin-Zwischenstufen in Paraffin.

Von den Barteln wurden nur Querschnitte angefertigt, von den Trichtern und Geruchsorganen Quer- und Längsschnitte, wobei die Schnittdicke zwischen 7-12 $\mu \mathrm{m}$ variiert wurde.

Für die färberische Darstellung der zu untersuchenden Gewebe kamen folgende Methoden zur Anwendung: Eisenhämatoxylin nach HeIdenhaIN, Hämatoxylin nach Delafield und die Kombination Säurealizarinblau-Anilinblau-Orange G. Die elektive Darstellung von Nerven, Sinnesknospen und Geruchsrezeptoren erfolgte durch die Silberimprägnationsmethoden nach Bodian (mit eigener Modifikation) und PALMGREN. Dabei erwies sich das Protargol von der Firma WINTHrop (USA) als vorzüglich geeignet.

\section{BEFUNDE}

\section{Morphologie \\ Kopf, Kopfanbänge und Kiemenregion}

In der Grundgestalt unterscheidet sich der Kopf von Rbinomuraena ambonensis kaum von dem anderer Muränen-Gattungen bzw. Arten. Der Schädel ist relativ schmal, wobei seine Breite von einem Bereich, der noch hinter der Orbitalregion liegt, bis zur Kopfspitze stetig abnimmt. Der Unterkiefer wird yom Oberkiefer leicht überragt. Von einer unterständigen Mundöffnung kann man jedoch kaum sprechen, da die Mundspalte eine enorme Ausdehnung hat; sie reicht bis weit hinter die Orbitalregion (Abb. 1, 2). Das bedeutet wiederum, daß das Kiefergelenk noch weiter caudad liegt, wie dies, wenn auch in geringerem Maße, bei anderen Muränen-Gattungen der Fall ist.

Von besonderem Interesse im Rahmen der vorliegenden Untersuchungen sind vor allen Dingen die verschiedenen „Kopfanhänge “ : ein fast spitzer Hautfortsatz, der 
von der Oberlippe ausgeht und der als "Rostrum" bezeichnet werden soll; zwei auffallend große, trichterförmige Hautlappen, zu denen die vorderen Öffnungen des Geruchsorgans bzw. deren Ränder ausgewachsen sind, sowie drei am vorderen Unterlippenrand entspringende Barteln (Abb. 1, 2). Insbesondere die beiden vom Oberlippenrand fast gerade nach vorn ragenden Hauttrichter, die an überdimensionierte Ohren erinnern, verleihen dem Kopf der Rbinomuraena ambonensis ein höchst bemerkenswertes Aussehen. Diesem Umstand verdankt Rbinomuraena wohl auch ihre Namen: Nasenmuräne bzw. Hasenmuräne.

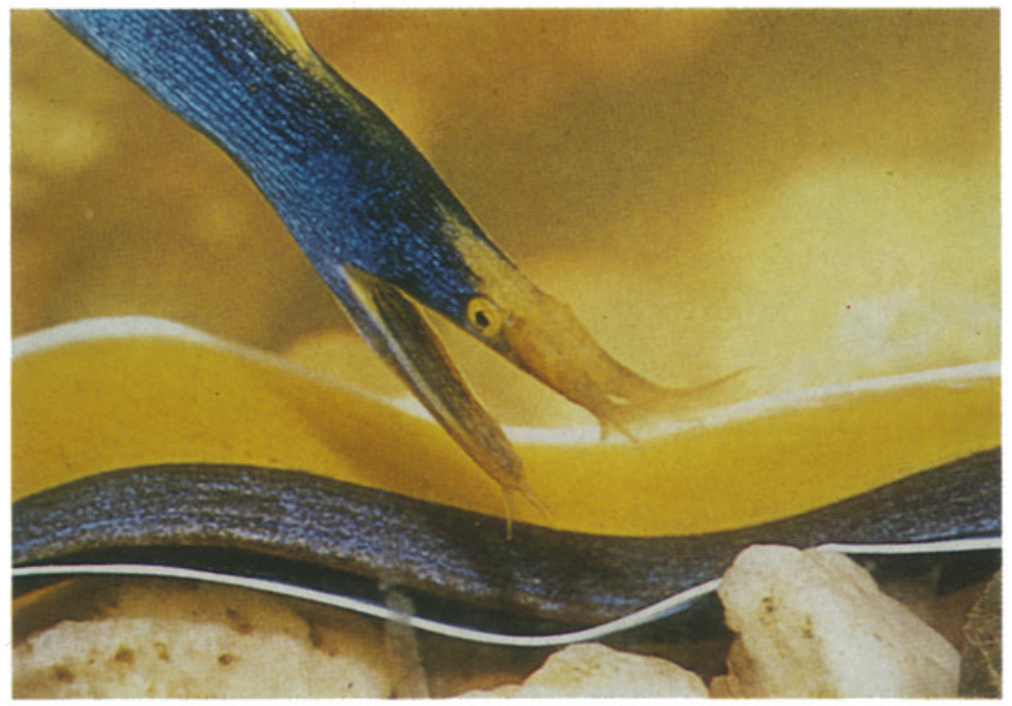

Abb. 1: Rhinomuraena ambonensis. Kopf, darunter Körperabsdhnitt hinter der Afterregion. Dorsaler Flossensaum gelb (Karotinoide) mit weißem Rand, Körper blau (Melanine), ventraler Flossensaum schwarz (Melanine) mit weißem Rand (Guanin?). ( 2:1)

Der Erwähnung wert ist schließlich noch die Gestaltung der Kiemenregion. Ahnlich wie bei den übrigen Muränen-Gattungen fehlt der Kiemendeckel. Dafür ist eine langgezogene Kiemenhöhle ausgebildet, die sich bis weit hinter die Occipitalregion ausdehnt. Sie öffnet sich durch einen relativ engen Spalt, dessen Ränder zu einer kurzen Röhre umgestaltet sind (Abb. 1,2). Die Durchströmung der Kiemenhöhle mit Atemwasser erfolgt durch peristaltische Bewegungen der muskulösen, lateralen Wand, wobei sich während jeder Kontraktionswelle die Hautränder im Bereich des Mundspaltenknickes einmal mediad zusammenlegen und dadurch den Kiemenraum rostrad verschließen.

\section{Geruchsorgan}

Während es bei mandhen anderen Fischarten bisweilen mit Schwierigkeiten verbunden ist, die Position des Geruchsorgans bzw. der Riechhöhle und ihrer Offnungen wegen ihrer geringen Ausdehnung ausfindig zu machen, gelingt es beim Geruchsorgan 
von Rbinomuraena ambonensis leicht, da es sich um eine in Größe und Gestaltung auffallende Bildung handelt (Abb. 2). Etwa $3 / 4$ des vor den Augen liegenden Kopfbereichs werden von den Riechsäcken und ihren Anhängen eingenommen, was den Gattungsnamen Rbinomuraena als äußerst zutreffend erscheinen läßt, wenn man einmal davon absieht, daß der Terminus "Nase" sowohl im anatomischen, wie auch im funktionellen Sinne für Knochenfische wenig glücklich ist.

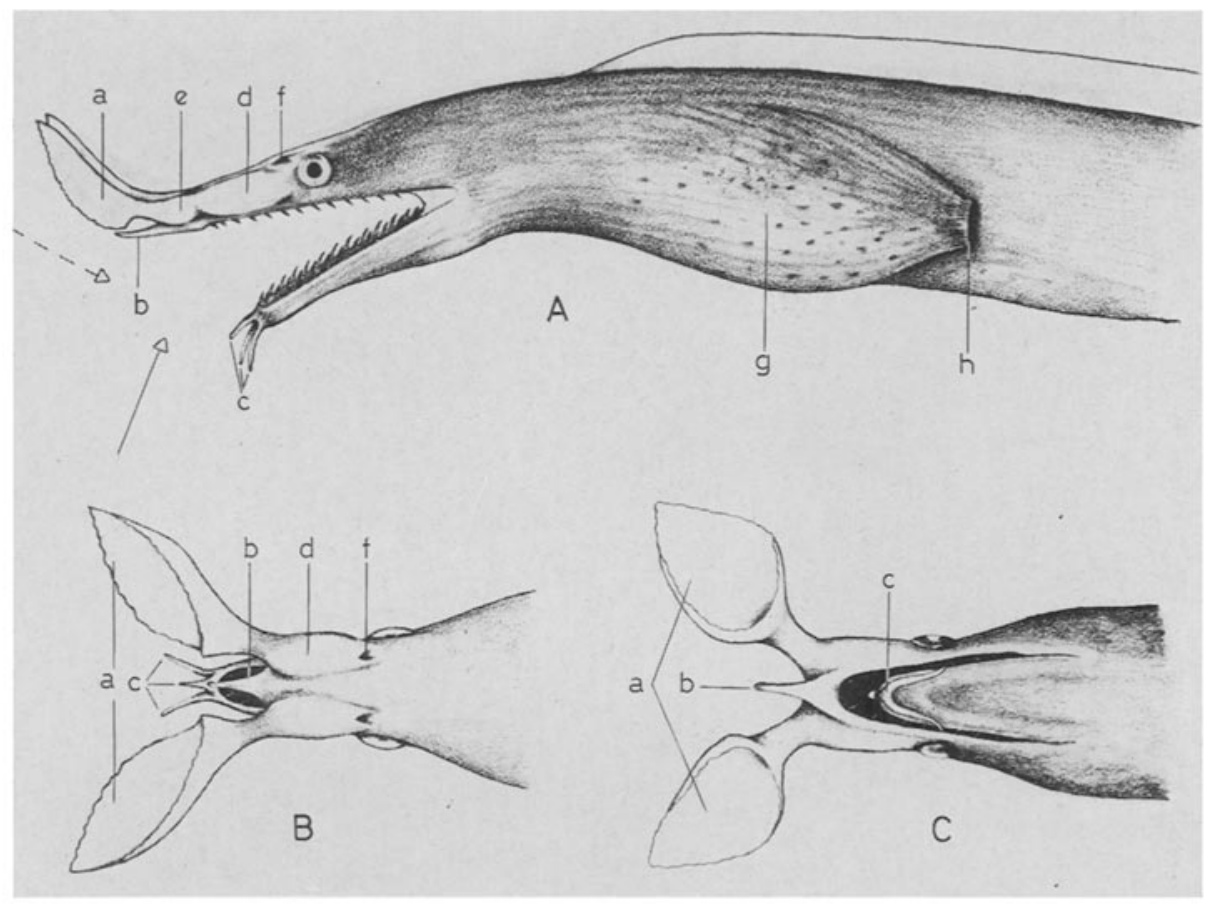

Abb. 2: Rhinomuraena ambonensis. A Vorderkörper von lateral, $B$ von dorsal (mit Blickrichtung entsprechend durchbrochenem Pfeil), $C$ von ventral (ausgezogener Pfeil). a trichterförmige Hautlappen; $b$ Rostrum; $c$ Mandibularbarteln; $d$ Riechsadk mit Geruchsorgan; $e$ Trichtermündung bzw. Vorderöffnung des Geruchsorgans; $f$ Hinteröffnung des Geruchsorgans; $g$ Kiemensack; $b$ Offnung des Kiemensacks. $(\sim 2: 1)$

Wie bei den anderen Muränen-Gattungen und überhaupt den meisten Vertretern der Anguilliformes sind die vorderen Offnungen des paarigen Geruchsorgans weit nach rostral an den Oberlippenrand der Kopfspitze verlagert. Die Ränder dieser vorderen Offnungen sind nun bei Rbinomuraena ambonensis in noch weit stärkerem Maße als bei den vergleichsweise erwähnten Formen ausgewachsen oder ausgezogen, und zwar nicht nur in der Länge, sondern auch in der Breite. Das Ergebnis sind zwei trichterförmige oder auch trompetenartige große Hautlappen, die in Verlängerung der Körperlängsachse, also fast gerade, weit über die Kopfspitze hinausragen. An der Basis verengt sich der Trichter zu einer Röhre mit relativ dicker Wandung, die sich dann unmittelbar in die große Riechhöhle fortsetzt (Abb. 1, 2). 
Die Riechhöhle selbst ist ein im Längsschnitt gestreckt ovaler, im Querschnitt runder Raum, der caudal bis nahe an den Augenvorderrand reicht und dabei in seiner Breite und Tiefe eine annähernd konstante Ausdehnung aufweist (Abb. 3). Er mündet durch eine relativ kleine, schlitzförmige, dicht vor dem Auge liegende Offnung nach außen. Das Riechhöhlendach ist über die gesamte Länge der Riechhöhle stark aufgewölbt. Die Ränder dieser Aufwölbung markieren dabei fast genau die seitliche Ausdehnung der Höhle (Abb. 2).

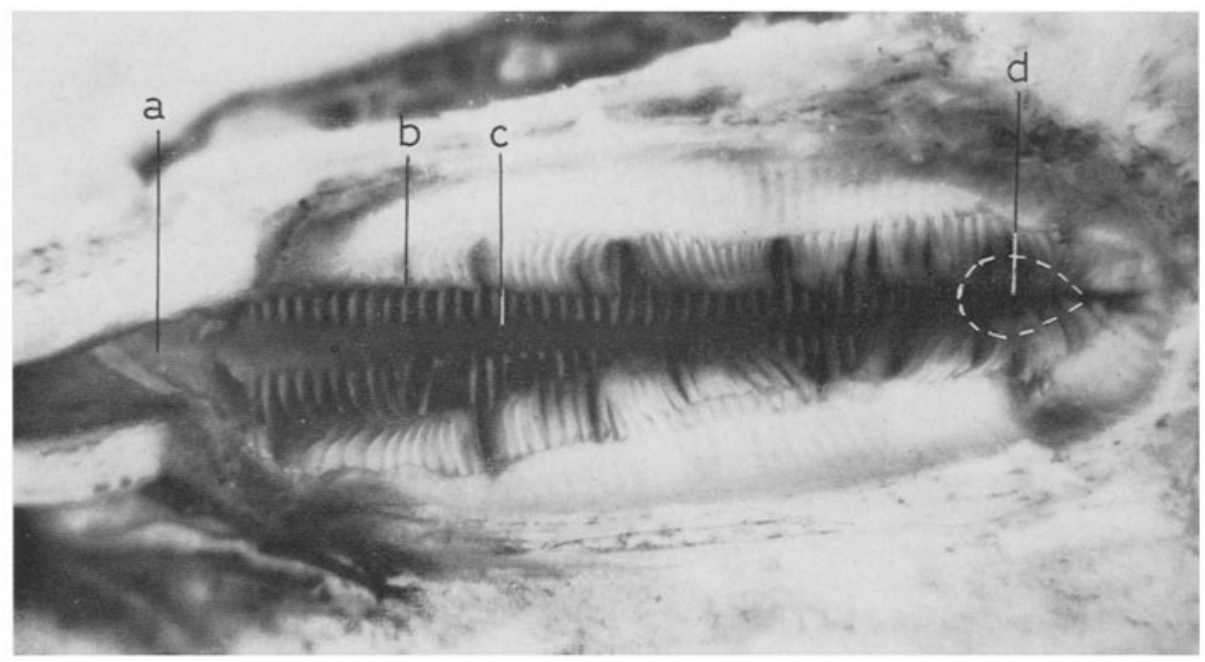

Abb. 3: Rhinomuraena ambonensis. Dorsal eröfnete Riechhöhle mit Riechlamellen. a Trichtermündung bzw. Vorderöffnung; $b$ dorsale, fingerförmige Abschnitte der Riechfalten; $c$ zylindrische Längsrinne (Strömungsrinne) zwischen den medianen Rändern der Riechfalten, darunter die Mittelraphe, an der die Riechfalten alternierend sitzen; d ungefähre Position der Hinteröffnung

Von besonderem Interesse am Geruchsorgan sind die Riechfalten. Diese lamellenartigen Hautduplikaturen, Erhebungen des Riechhöhlenbodens bzw. der Wände, sind bei Rbinomuraena ambonensis in einer extrem hohen Zahl ausgebildet, die selbst die Zahl der Riechfalten von Anguilla anguilla übertrifft (Abb. 3, 4). Die Auszählung der Falten bei den zwei für diese Untersuchung zur Verfügung stehenden Tieren ergab folgende Werte:

Tabelle 1

Anzahl der Riechfalten im Geruchsorgan von Rbinomuraena ambonensis

\begin{tabular}{|cccccc|}
\hline \multirow{2}{*}{ Tier Nr. } & \multicolumn{2}{c}{ Linkes Organ } & \multicolumn{2}{c|}{ Rechtes Organ } & $\Sigma$ \\
& median & lateral & median & lateral & \\
\hline 1 & 55 & 56 & 53 & 55 & 219 \\
2 & 47 & 46 & 44 & 45 & 182 \\
\hline
\end{tabular}

Diese Riechfaltenzahlen zeigen ganz eindeutig, daß bei Rhinomuraena ambonensis eine sehr eindrucksvolle Vergrößerung des Riechfeldes vorliegt, ganz entspre- 
chend den Verhältnissen wie sie vom Aal schon bekannt sind (TEICHMAnN 1954 etc.). Im Unterschied zu Anguilla anguilla sind hier jedoch die Riechfalten noch wesentlich gedrängter angeordnet (Abb, 3, 4). Bei einem Aal mit der Kopflänge, die derjenigen der hier bearbeiteten zwei Muränen entspricht, stehen bei einer Gesamtlänge des Riechsackes von ca. 6-8 $\mathrm{mm}$ nur etwa höchstens 15-20 Falten (bzw. die doppelte Anzahl, wenn man die doppelte Faltenreihe einer Kopfseite heranzieht). Bezogen auf die gleiche Kopflänge stehen also bei Rbinomuraena ambonensis dreimal soviel Riechfalten wie bei Anguilla anguilla.

Diese extrem hohe Riechfaltenzahl muß man in Zusammenhang mit dem Anordnungsmuster der Falten sehen. Unschwer läßt sich erkennen, daß dieselben nach dem 900-Typ (Holl 1965) angeordnet sind. Das heißt, die caudalen, ältesten Lamellen zeigen eine radiäre Ausrichtung ihrer Achse, die daran anschließenden Falten hingegen

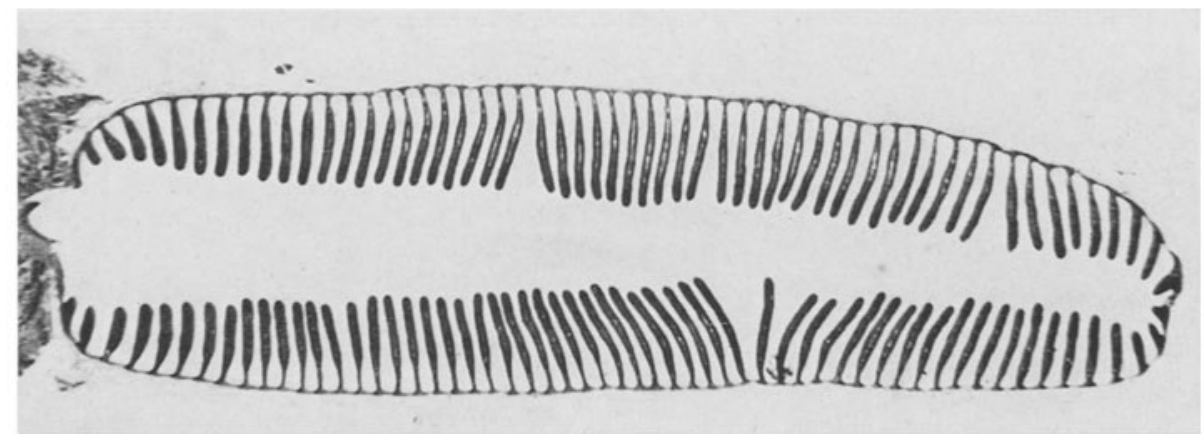

Abb. 4: Rhinomuraena ambonensis. Längsschnitt durch das Riechfaltensystem in Höhe der Rinnenachse

stehen parallel zueinander. Dieser Anordnungsweise zufolge liegt bei Rbinomuraena ambonensis ein doppelreihiges Faltensystem vor, bei dem die einzelnen Falten der beiden Reihen entlang einer geraden, sich rostrad verbreiternden sog. Mittelraphe ansitzen.

Da die gesamte Distanz zwischen Augenvorderrand und Oberlippenrand von der Riechhöhle eingenommen wird, so entsteht - zumal die Bildungszone für das Auswachsen neuer Falten nicht nur während der Jugendentwicklung tätig ist - ein extrem gestrecktes polylamelläres System (Abb. 3, 4). Daß die Faltenbildungszonen während des gesamten Lebens weitere, junge Falten liefern, kann angenommen werden, da das kleinere, offenbar jüngere Tier wesentlich weniger Falten aufweist als das größere, ältere Tier. Dabei ist zu berücksichtigen, äaß die Zuwachsrate mit zunehmendem Alter erfahrungsgemäß abnimmt, etwa so wie es von Anguilla anguilla bekannt ist. Eine verbindliche Angabe über die Gesamtzahl der Riechfalten bei extrem alten Tieren kann nicht gemacht werden, da keinerlei Beobachtungen oder Untersuchungen über Entwicklung, Wachstum und Alter von Rbinomuraena ambonensis vorliegen.

Unterzieht man die Auswuchszonen der einzelnen „fertigen“ Falten, die nicht identisch sind mit den Neubildungszonen für junge Falten, der näheren topographi- 
schen Analyse, so läßt sich feststellen, daß diese sich weit über den Bereich des Riechhöhlenbodens bis hin zum Ansatz des Riechhöhlendaches erstrecken. Von hier aus ragt jede einzelne Falte mit einer fingerförmigen Projektion in die Riechhöhle, wobei der Abstand zum daruberliegenden Riechhöhlendach gering ist (Abb. 3). An der Mittelraphe inserieren die einzelnen Falten alternierend in fast regelmäßiger Weise (Abb. 11).

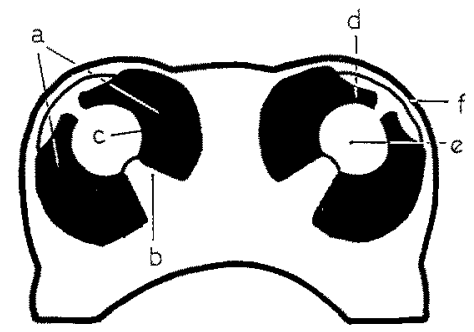

A

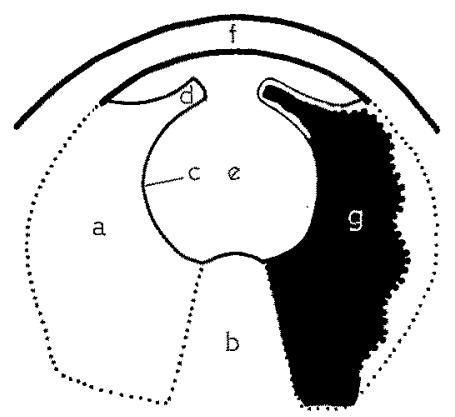

B

Abb. 5: Rhinomuraena ambonensis. A Geruchsorgan frontal mit Oberkiefer, zur Demonstration der stark geneigten Querachse des Organs und der Riechlamellenform (schwarz) und -größe (schematisch), B einzelnes Organ frontal, Riechfeldausdehnung rechts (schwarz). a Riechfalte; $b$ Mittelraphe; $c$ innerer bzw. medianer Faltenrand; $d$ dorsaler, fingerförmiger Abschnitt der Riechfalte; $e$ Längsrinne; $f$ Riechhöhlendach; $g$ Riechfeld

In ihrer Gestalt und auch Größe sind die einzelnen Riechfalten fast identisch. Dabei ist besonders bemerkenswert, daß der innere, freie Rand jeder Falte von der Spitze des fingerartigen Fortsatzes bis zum Ansatz an der Mittelraphe einem annähernd halbkreisförmigen Bogen entspricht (Abb. 5). In ihrer Gesamtheit betrachtet, bilden so die inneren Ränder der zahlreichen Falten jeder Seite die Grenze eines über der Mittelraphe liegenden, sich von den caudalen Falten bis hin zur Trichtermündung erstreckenden, zylindrischen Hohlraums, der als die direkte Fortsetzung des Trichtereinlasses anzusehen ist (Abb. 3, 4). Auch beim Aal und anderen Arten mit verlängertem $90^{\circ}$-Faltensystem findet sich zwischen Riechhöhlendach und den dorsalen Rändern der Falten ein mehr oder weniger großer Raum, der jedoch relativ niedrig bzw. flach ist und durchaus nicht immer - (wohl aber bei Anguilla anguilla) (Holl 1965) die 
unmittelbare Fortsetzung der vorderen Offnung darstellt. Caudal mündet dieser zylindrische Raum in die hintere Offnung der Riechhöhle.

Wie an Hand von Strömungsexperimenten mit Tuscheteilchen nachgewiesen werden konnte, fungiert der von den freien Riechfaltenrändern umschlossene zylindrische Raum als Kanal für den Transport der Hauptmenge des durch die Trichtermündung eingeströmten Wassers, das schließlich durch die hintere Öffnung die Riechhöhle verläßt. Während der Wasserpassage durch diesen Kanal werden infolge von Staueffekten der dorsalen und medianen Faltenränder laminare Randströmungen in die interla-

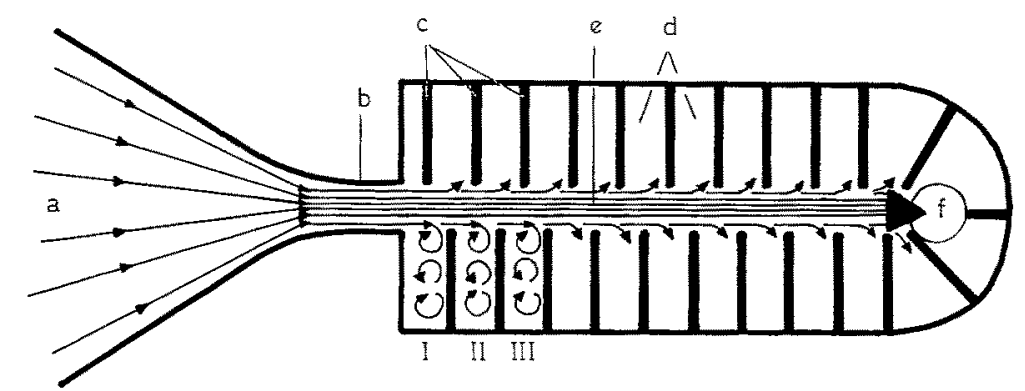

Abb. 6: Rhinomuraena ambonensis. Geruchsorgan als physikalisches System, das die Passage des Duftwassers (Ventilation) idealisiert zeigt. Richtung der Strömungen durch Pfeile markiert. Weit auseinander liegende Strömungslinien = langsame Fließgeschwindigkeit, dicht ziehende Linien $=$ größere Fließgeschwindigkeit. $a$ Trichteröffnung; $b$ Trichtermündung; $c$ Riechlamellen; $d$ interlamelläre Räume; e zylindrische Längsrinne; $f$ Hinteröffnung; in den

interlamellären Räumen I, II, III sind die auftretenden Mikroturbulenzen eingezeichnet

mellären Räume abgezweigt. Im Bereich dieser Spalten kommt es dann zu zahlreichen, winzigen Turbulenzen (Abb. 6), die für die Heranführung der Duftmoleküle an die Riechschleimhaut wichtig sind.

In diesem Zusammenhang erscheint es notwendig, auch auf die Frage nach dem Austausch des Wassers zwischen den Falten einzugehen. Dieser Austausch erfolgt wie weitere Versuche mit Tuscheteilchen an der eröffneten Riechhöhle zeigten - in der Weise, daß von der im Längskanal herrschenden, caudal gerichteten Strömung Wasserportionen mitgerissen werden (Abb. 6).

Als relativ effektiv für den Wasseraustausch zwischen den Riechfalten erwies sich der Schlag der Kinocilien, mit denen die Flimmerzellen des Riechepithels besetzt sind.

\section{Funktionelle Morphologie der Trichter}

Wie die Beobachtungen am lebenden Tier zeigten, ist die Funktion der beiden Trichterorgane bei der Versorgung des Geruchsorgans mit Duftwasser ${ }^{1}$ eine doppelte. Zunächst einmal haben die Trichter die Aufgabe, das sich beim langsamen oder schnellen Vorwärtsschwimmen des Fisches an den Innenflächen stauende Wasser in die Offnung an der Trichterbasis hineinzulenken. Wenn man die Größe der Innenflächen

1 Als Duftwasser wird das Wasser bezeichnet, das die Duftinformationen trägt. 
in Relation setzt zur Geschwindigkeit des Fisches, so ergeben sich ganz beachtliche Werte für den auftretenden Staudruck. Davon aber hängt es ab, wie groß die Wassermenge ist, die pro Zeiteinheit die Riechhöhle passiert. Zum anderen erhebt sich die Frage nach der Duftwasserversorgung des Geruchsorgans bei Ruhestellung des Tieres. Immerhin ist Rbinomuraena ambonensis, wie im übrigen wohl die meisten MuränenGattungen, ein Litoralbewohner.

Sie dürfte sich, wie es für die Vertreter der Gattung Muraena gilt, in Hohlräumen zwischen größeren Steinen oder in Lücken von Korallenbänken bei relativ geringer Bewegungsaktivität aufhalten. Dann aber ist es für den Fisch bedeutungsvoll, „alarmierende" Duftinformationen zu erhalten.

Auch hier hilft die Beobachtung des lebenden Tieres im Aquarium und der Versuch mit Tuscheteilchen weiter. Dabei läßt sich folgendes feststellen: Die Atemtechnik von Rhinomuraena (und natürlich auch der anderen Muränen-Gattungen) ist völlig verschieden von der Atembewegung der meisten anderen Knochenfische. Sie erfolgt, wie schon erwähnt, durch peristaltische Bewegungen der lateralen Wand des Kiemenraumes. Aus dieser rhythmischen Bewegung des Kiemenapparates resultiert eine deutliche Wasserströmung von allen Seiten in Richtung auf den Mundspalt. Da die beiden Trichterorgane im oberen Bereiche dieser Strömung liegen, gelangt ein Teil des so bewegten Wassers durch die Trichtermündung in die Riechhöhle und schließlich wieder durch die hintere Offnung nach außen. Dabei wird dann das Geruchsorgan, im Gegensatz zur Situation beim Vorwärtsschwimmen, in leicht thythmischer Weise ventiliert.

\section{His tologie \\ Trichter}

Die im morphologischen Abschnitt als Hautlappen bezeichneten trichterförmigen Auswüchse des Randes der vorderen Riechöffnungen zeigen einen einfachen histologischen Bau, an dem - von den Gefäßen und Nerven einmal abgesehen - zwei Gewebekategorien beteiligt sind: Epithel und Bindegewebe.

Das Epithelgewebe ist mehrschichtig und bildet die äußere und innere Trichterwand. Der Zentralteil besteht aus Bindegewebe (Abb. 7). Dabei ist zu bemerken, daß das Epithel der Trichterinnenseite sich aus nur 3-4 Zellagen zusammensetzt, während das Epithel der Außenseite 5-8 Zellagen aufweist, also wesentlich dicker ist. Die Epithelien der Außen- und Innenseite der Trichter haben eine weitgehend gleichbleibende Didke (Didke des Epithels der Trichterinnenseite: 10-15 $\mu \mathrm{m}$, Dicke des Epithels der Außenseite: 30-35 $\mu \mathrm{m}$ ), Auswüchse oder Erhebungen sind lichtmikroskopisch nicht nachweisbar. Bei den verschiedenen Zellagen beider Epithelien handelt es sich im einzelnen um eine periphere Reihe von Abschlußzellen, ein bis mehrere Lagen von $Z$ wischenzellen, sowie eine basale Reihe von Bildungszellen, die einer dicken $\mathrm{Ba}$ salmembran aufsitzen (Abb. 7, 8, 9).

Zwischen den Epithelzellen liegen in unregelmäßiger Verteilung Sekretzellen. Insgesamt betrachtet sind diese Sekretzellen, bei denen es sich wohl um Schleimbecherzellen der üblichen bei Fischen bekannten Art handeln dürfte, im Epithel der Trichteraußenseite deutlich zahlreicher vertreten als im Epithel der Innenseite. 
Zum Bindegewebe hin ist das Epithel an zahlreichen Stellen zu kleinen Vorsprüngen aufgeworfen, die spitz zulaufen. In anderen Bereichen verläuft die Grenze des Epithels zum Bindegewebe dagegen annähernd geradlinig. Wie nun die histologische

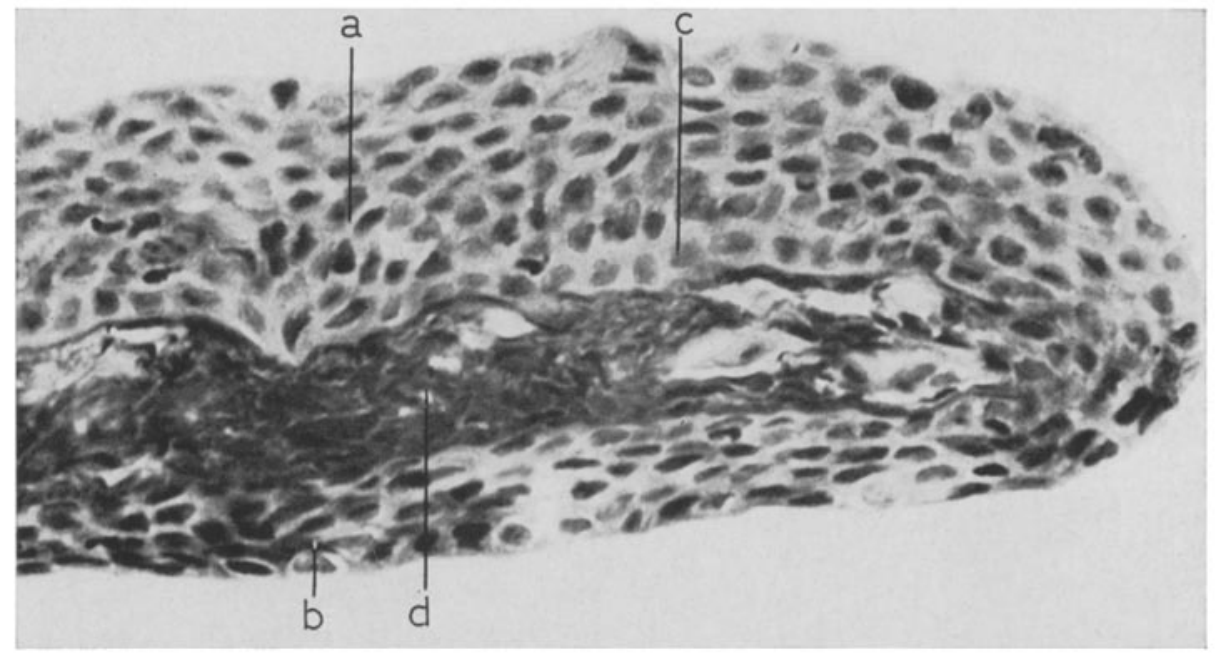

Abb. 7: Rbinomutaena ambonensis. Querschnitt durch die Trichterwand in der Nähe des Randes. $a$ Epithel der Trichteraußenseite; $b$ Epithel der Innenseite; $c$ Epithelbildungszellen; $d$ Bindegewebe. $(650: 1)$

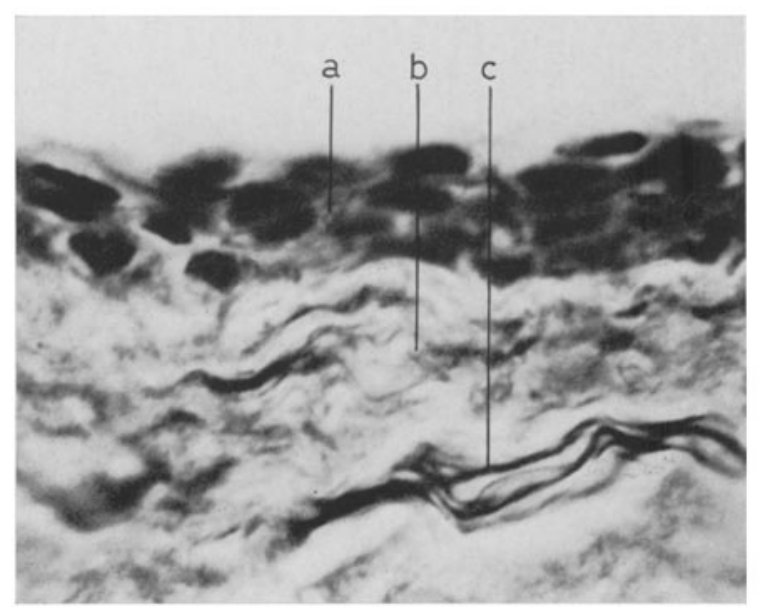

Abb. 8: Rbinomuraena ambonensis. Ausschnitt der Trichterwand, Innenseite mit Nervenbündel im Bindegewebe. $a$ Epithel; $b$ Bindegewebe; $c$ Nervenbündel. (Versilberung nach Bodian; $1200: 1$ )

Untersuchung dieser „Auswüchse“ (Darstellung durch Silberimprägnation) zeigte, treten hier öfter feine Nervenfaserbündel vom Epithel ins Bindegewebe (Abb. 9). Solche Faserbündel finden sich aber auch dort, wo die basale Epithelgrenze glatt verläuft. 
Außerdem konnte nachgewiesen werden, daß häufig an der Grenze zwischen den basalen Bildungszellen des Epithels und den übrigen Epithelzellen locker gebündelte oder einzelne Nervenfasern verlaufen. In verschiedenen Fällen erwiesen sich die oben erwähnten, ins Bindegewebe ziehenden, Faserbündel als die Fortsetzung dieser intraepithelialen Nervenfasern (Abb. 9).

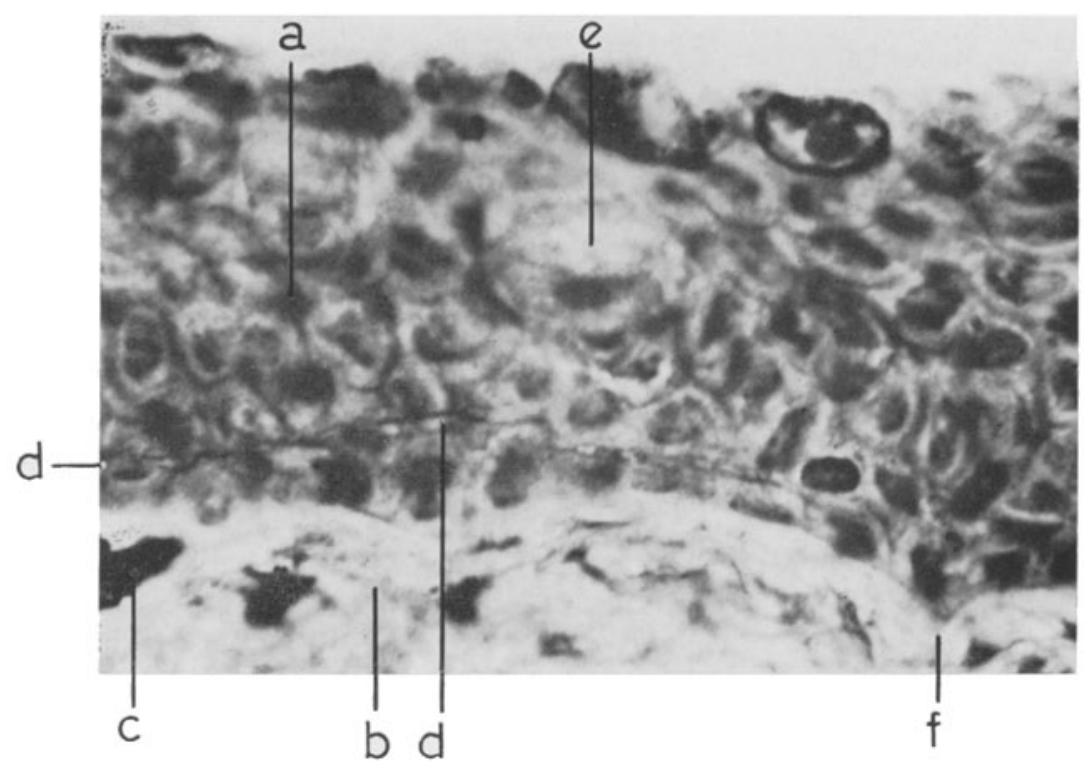

Abb. 9: Rhinomuraena ambonenszs. Ausschnitt der Trichterwand, Außenseite. Epithel mit feinem, intraepithelialem Nervenfaserbündel, das zur Epithelprojektion zieht. a Epithel; $b$ Bindegewebe; $c$ Pigmentzelle; $d$ intraepitheliales Faserbündel, das an der Grenze zwischen Epithelbildungszellen und den darüberliegenden Zellen verläuft (mit mehreren bläschenförmigen Anschwellungen (Pfeile); e Schleimzelle; $f$ Epithelprojektion zum Bindegewebe. (Versilberung nach BodraN; $1200: 1$ )

Nicht selten finden sich an den Fasern bläschenförmige Anschwellungen in sehr unregelmäßiger Anordnung, die nur schwach imprägniert sind (Abb. 9). Hier wird erst die elektronen-mikroskopische Untersuchung eine Klärung bringen können. Derartige Faserbildungen konnten nur im Epithel der Trichteraußenseite gefunden werden. Wahrscheinlich sind sie auch im Epithel der Innenseite vorhanden; nur dürfte ihre Zahl und Ausdehnung geringer sein.

Typische freie Nervenendigungen wurden im Epithel ebensowenig nachgewiesen wie einzelne Sinneszellen.

Auffallend ist dagegen die Existenz von Sinnesknospen im Epithel der Trichter. In Gestalt und Aufbau erinnern diese Organe stark an Geschmacksknospen (Abb. 10). Sie sind jeweils aus mehreren Stützzellen (bzw. indifferenten Zellen) und Sinneszellen aufgebaut, deren Kerne im basalen und mittleren Bereich der Knospe liegen. Der periphere Abschnitt der Knospe ist kernfrei und iiberragt um 5-10 $\mu \mathrm{m}$ die Epitheloberfläche. Anders als bei Geschmacksknospen, deren Basis gewöhnlich direkt dem 
Bindegewebe aufsitzt, sind die erwähnten Sinnesknospen vom Bindegewebe durch eine bis mehrere Lagen von Epithelzellen isoliert. Über die Innervierung der Sinnesknospen gaben die Versilberungen wenig Aufschluß. Nur in einem Falle traten feine nervöse Endverästelungen bei einer Sinnesknospe in Erscheinung.

Die Anzahl dieser Sinnesknospen im Epithel des Trichters ist gering, ihre Dichte liegt weit unter der von Geschmacksknospen etwa in den Epithelien der Mundhöhle von Muraena oder auf Barteln anderer Fischarten (beispielsweise bei Siluriden, Gadiden oder Cypriniden).

Den größten Anteil am Aufbau des Trichters hat die bindegewebige Zentralschicht. Ihre Dicke nimmt von ca. $0,5 \mathrm{~mm}$ im Bereich der Trichtermündung bis auf etwa $20 \mu \mathrm{m}$ am Trichterrand kontinuierlich ab. Die Faserarchitektonik der bindegewebigen Elemente, die zu kleineren und größeren Bündeln zusammengefaßt sind, zeigt nur insofern eine gewisse Einheitlichkeit, als eine innere, dickere Zone straffe Faserbündel aufweist, die vornehmlich in der Trichterlängsachse ausgerichtet sind,

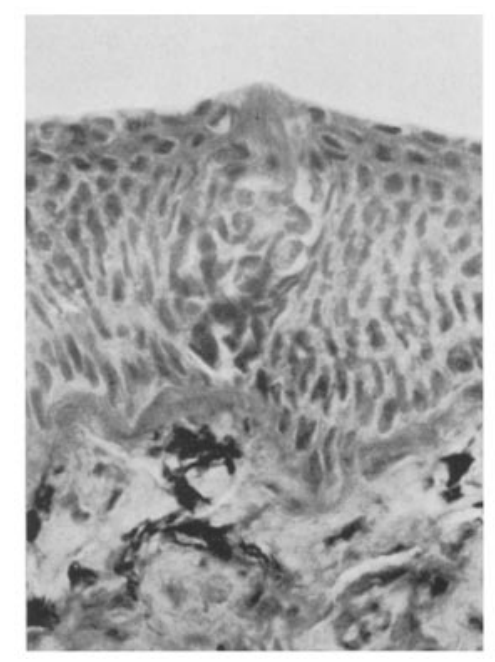

Abb. 10: Rbinomuraena ambonensis. Sinnesknospe im Epithel des Rostrums (unterscheidet sich nicht von den Sinnesknospen des Trichterepithels). (500:1)

während die feineren Bündel der äußeren Zone (in der Nähe des Epithels) senkrecht dazu verlaufen. Lichtmikroskopische Färbungen lassen auf einen großen Kollagenanteil schließen. Besondere Unterschiede in der histologischen Struktur etwa zum Bindegewebe der Riechhöhlenhaut waren nicht zu erkennen. Auffallend ist, daß der Trichter keine Muskulatur aufweist.

Eingebettet im Bindegewebe liegen feinere Gefäße sowie insbesondere in Epithelnähe Kapillaren. Dieses System stammt offenbar von einem Gefäß, das im Dach der Riechhöhle verläuft und im Bereich der Trichtermündung verzweigt.

Während die Haut von Rbinomuraena ambonensis in allen anderen Körperregionen in reichem Maße Pigmentzellen aufweist, liegen im Bindegewebe des Trichters 
nur vereinzelt Melanophoren, und zwar dicht unter der Basalmembran. So kommt es, daß die Trichter fast transparent erscheinen.

Besonderes Interesse verdient schließlich noch die Innervierung der Trichter, da sich hier unter Umständen ein Anhaltspunkt für die Funktion dieses Organs ergibt. Mit Hilfe von Versilberungen gelang es, die Nervenfaserbündel selektiv darzustellen und so einen Überblick über ihr System zu gewinnen. Zunächst ist bemerkenswert, $\mathrm{da} B$ das Bindegewebe der Trichter zahlreichere und zum Teil auch umfangreichere Nervenfaserbündel (Abb. 9) enthalten als die Haut irgendeines anderen Körperabschnittes. An Längsschnitten durch die Wand der Trichtermündung war außerdem ersichtlich, daß mehrere dicke Nervenbündel, die dem Trigeminussystem angehören, den größten Teil der Nervenfasern aus dem Trichter sammeln. Über den zentralen Verlauf und Anschluß weniger kleinerer Faserbündel, die möglicherweise den spärlich vorhandenen Sinnesknospen zuzuordnen sind, gab es keine Hinweise für eine verbindliche Aussage. In Frage kämen jedenfalls die dorsalen Rami des Nervus facialis und Nervus trigeminus (V,1 $+\mathrm{VII}, 1)$.

Auf Grund der oben erwähnten relativ starken Innervierung der Trichter wäre durchaus eine größere Zahl von Endorganen zu erwarten, eventuell Sinnesorgane, die im Bindegewebe liegen könnten. Außerdem muß betont werden, daß nur in wenigen Fällen Beziehungen zwischen den feinen intraepithelialen Fasergruppen und dickeren Faserbündeln im Bindegewebe gefunden wurden. Nach den vorliegenden Untersuchungen gibt es jedoch keine Anhaltspunkte dafür, daß außer den beschriebenen epithelialen Sinnesknospen sowie intraepithelialen Einzelfasern oder Fasergruppen weitere rezeptorische Elemente im Bereich der Trichter existieren.

\section{Riechböblenwand und Riechfalten}

Die Riechhöhle - damit sind gemeint: das Riechhöhlendach, die Bereiche zwischen den lateralen und ventralen Riechfaltenansätzen sowie das rostrale Ende der Riechhöhle (also der Übergang zur Trichtermündung bzw. zur vorderen Offnung) ist von einem Epithel überzogen, das sich hinsichtlich seines Aufbaus und der Art der Zellen nur unwesentlich von dem des Trichters unterscheidet. Die Dicke dieses Epithels variiert zwischen 7 und $15 \mu \mathrm{m}$, liegt also etwa zwischen jener des äußeren und inneren Trichterepithels. Schleimzellen treten, mit Ausnahme des dorsalen Teils der Mittelraphe, nur selten auf.

Im Bereich der lateralen, ventralen und dorsalen Faltenansätze geht dieses indifferente Epithel kontinuierlich in das Riechepithel der Falten über. Bei den ventralen Faltenansätzen ist der Übergang zum Riechepithel wesentlich schroffer. Besonders deutlich läßt sich der Charakter des Riechepithels an der erheblichen Dickenzunahme und an dem Auftreten von Flimmerhärchen an der Peripherie des Riechepithels erkennen.

In seinem Bau erinnert das Riechepithel von Rhinomuraena ambonensis stark an die entsprechenden Verhältnisse bei Anguilla anguilla (HoLl 1965, LAIBACH 1937). Neben Rezeptoren treten auf: Stützzellen, Flimmerzellen, basale Bildungszellen und Schleimbecherzellen. Der Sekretzellen-Typ, wie er vom Aal bekannt ist (Hour 1965) war nicht nachweisbar. Das Riechepithel weist, wie auch das des Aals, nur eine relativ 


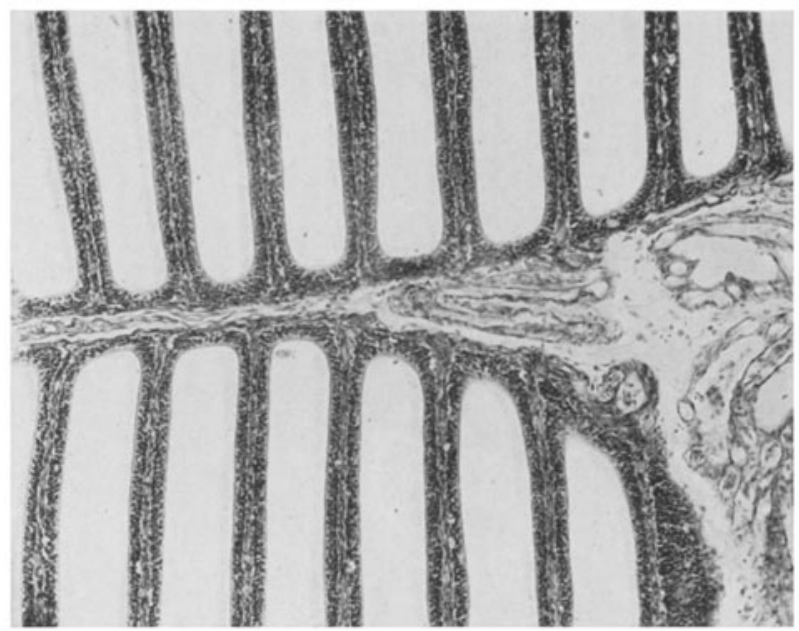

Abb. 11: Rhinomuraena ambonensis. Ausschnitt des Riechfaltensystem mit Mittelraphe. Alternierender Ansatz der Falten. (50:1)

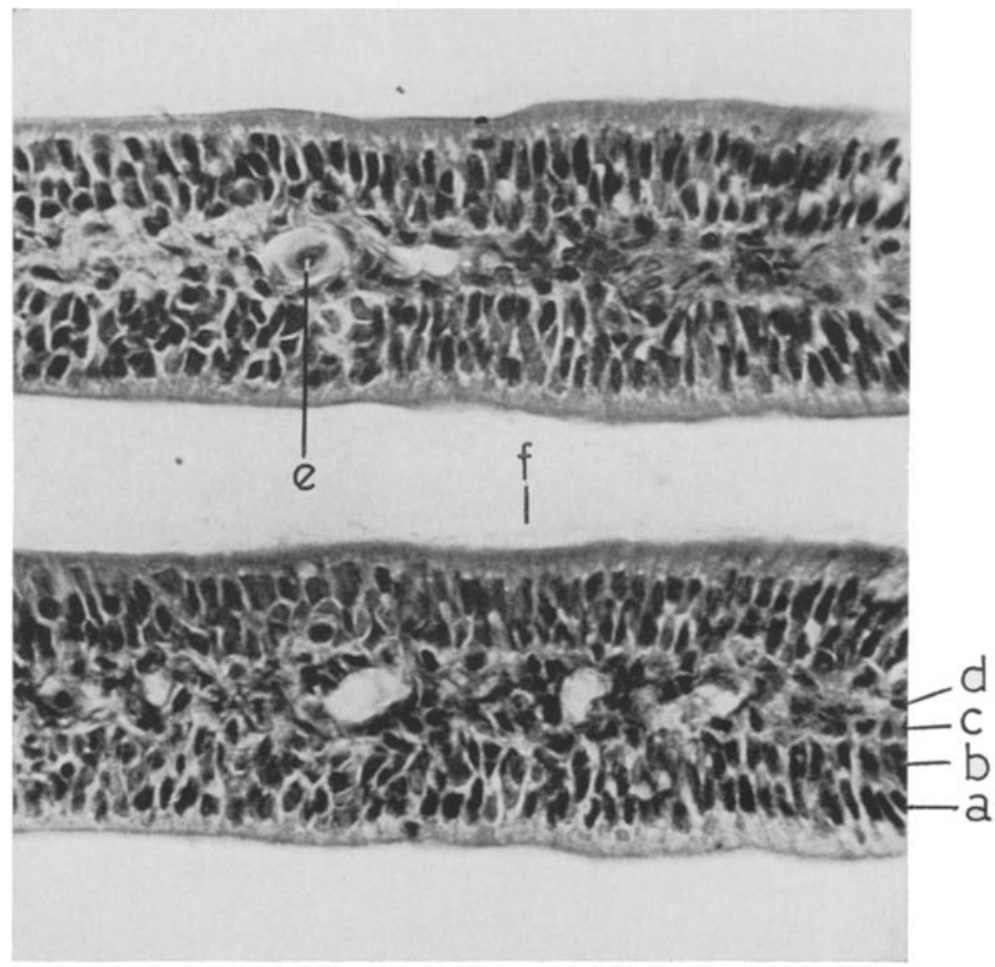

Abb. 12: Rbinomuraena ambonensis. Zwei Riechfalten im Schnitt. a Kerne der Flimmerzellen; $b$ Kerne der Rezeptoren; $c$ Kerne der Basalzellen; $d$ Bindegewebe; $e$ Gefäß $f f$ Flimmerhärchen. $(500: 1)$ 
geringe Dicke auf im Vergleich mit den entsprechenden Dimensionen beim Flußbarsch und Hecht (Riechepitheldicke Rbinomuraena ambonensis: 20-25 $\mu \mathrm{m}$, Anguilla anguilla: 35-50 $\mu \mathrm{m}$, Perca fluvietilis: 60-70 $\mu \mathrm{m}$, Esox lucius: 95-110 $\mu \mathrm{m}$ ). Die spindelförmigen Rezeptoren sind entsprechend gedrungener, insbesondere gilt das für den keineswegs sehr schlanken, peripheren Fortsatz (Abb. 13). Verschiedene morphologische Typen von Rezeptoren existieren nicht.

Im Hinblick auf die Verteilung der Kerne innerhalb des kontinuierlichen Riechepithels finden wir relativ einfache und einheitliche Verhältnisse (Abb. 12). Im Abstand von ca. 5-1 $\mu \mathrm{m}$ von der Epithelperipherie liegt eine Einzelreihe länglicher, dicht angeordneter Kerne, die den Flimmerzellen angehören. Unmittelbar darunter folgen in unregelmäßiger Anordnung die ebenfalls länglichen Rezeptorenkerne und

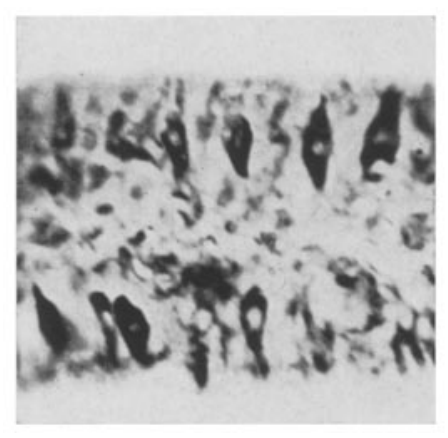

Abb. 13: Rbinomuraena ambonensis. Riechepithelausschnitt mit elektiv dargestellten keulenförmigen Rezeptoren. Kerne hell. (Versilberung nach Bodian; 600:1)

schließlich dicht über der Basalmembran eine lockere Reihe kleinerer Kerne, die den Bildungszellen zuzuordnen sind. Nur vereinzelt finden sich Kerne von Stützzellen (siehe HoLx 1965).

Außer diesen vier wichtigsten Elementen des Riechepithels sind noch die Schleimbecherzellen zu erwähnen, die in größeren Portionen (10-20) im Bereich der medianen Faltenränder auftreten, in allen anderen Faltenbezirken dagegen nur vereinzelt. Bei Auszählung der Rezeptorenkerne ergibt sich als durchschnittliche Rezeptorendichte ein Wert von knapp unter $30000 \mathrm{R} / \mathrm{mm}^{2}$.

Die Flimmerzellen des Riechepithels sind dicht besetzt mit langen $(15 \mu \mathrm{m})$ Flimmerhärchen. Soweit dies aus dem lichtmikroskopischen Bild ersichtlich ist, sind die Flimmerhärchen mit den wesentlich kürzeren Sinneshärchen, die aus niedrigen Vesiculae olfactoriae der Rezeptoren ragen, verklebt.

Schließlich ist noch zu klären, inwieweit das Riechepithel die Riechfalten bedeckt, bzw. welche Abschnitte der Falten von Riechepithel überzogen sind. Der dazu notwendige Vergleich aller Schnitte der Serien ergab, daß die Riechfalten bei Rbinomuraena ambonensis in noch größerem Ausmaß als bei Anguilla anguilla echtes Riechepithel tragen. Nur kleine Teile sind mit indifferentem Epithel überzogen, außer den Bereichen, die das bereits vereinzelt Rezeptoren enthaltende Ubergangsepithel aufweisen. Frei von Riechepithel sind eine schmale Zone des dorsalen Faltenrandes, Teile des 
inneren Faltenrandes, Teile der basalen Faltenansätze, die lateralen und die dorsalen Faltenansätze. Alle übrigen Partien einer Riechfalte, auch der ventrale Faltenansatz tragen Riechepithel (Abb. 5b). Dabei ist zu erwähnen, daß der Bereich des Übergangsepithels am lateralen Faltenansatz am größten ist.

\section{Rostrum und Mandibularbarteln}

Im histologischen Aufbau unterscheiden sich das Rostrum der Oberlippe und die drei Unterkieferbarteln nur unwesentlich. Etwas verschieden ist dagegen die Dicke der Epithelien, die bei den drei Barteln zwischen 20-50 $\mu \mathrm{m}$ beträgt, beim Oberkiefer-Rostrum dagegen $50-80 \mu \mathrm{m}$.

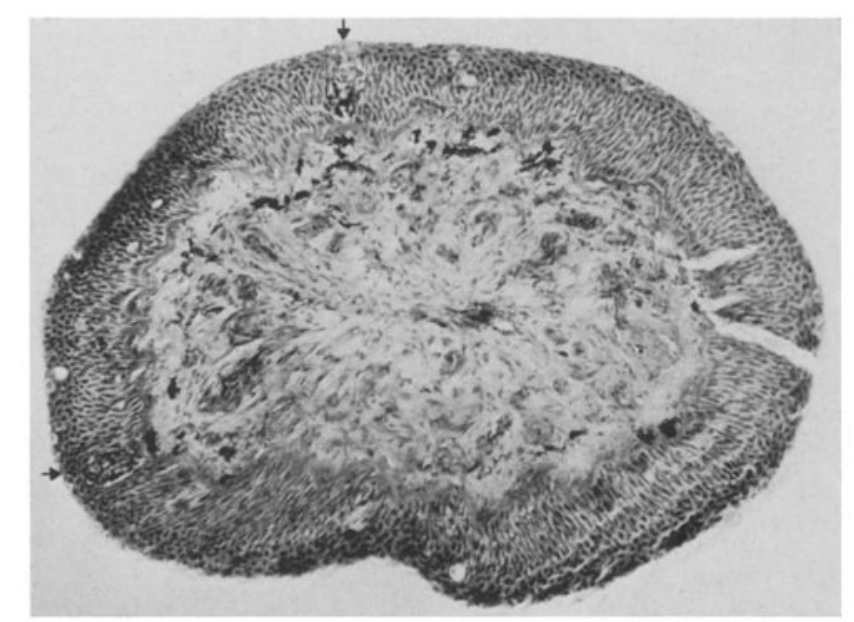

Abb. 14: Rbinomuraena ambonensis. Querschnitt durch das Rostrum. Pfeile zeigen auf angeschnittene Sinnesknospen. (120:1)

In allen Fällen ist das Epithel mehrschichtig. Es zeigt eine ähnliche Anordnung seiner Zellen wie das Trichterepithel. Innerhalb des Epitheis sitzen vereinzelt Schleimbecherzellen. Gegen das Bindegewebe findet sich als Abgrenzung eine relativ dicke Basalmembran. Ihr ungerader Verlauf entspricht den zahlreichen epithelialen Vorsprüngen zum Bindegewebe (Abb. 14).

Uberraschenderweise waren auch im Epithel des Rostrum und der Mandibularbarteln Sinnesorgane nachweisbar. Pro Querschnitt sind im Rostrum mindestens drei solcher Sinnesknospen getroffen, auf den Mandibularbarteln zeigt sich nur auf etwa jedem dritten bis fünften Schnitt ein solches Sinnesorgan. Dabei ist der terminale Teil des Rostrum - ebenso wie die Bartelspitzen - auf einer Länge von ca. $1 \mathrm{~mm}$ frei von Sinnesknospen. Nach den lichtmikroskopischen Untersuchungen handelt es sich hier um die gleichen Organe, wie sie vereinzelt auch im Epithel der Trichter registriert wurden (Abb. 10, 14). 
Den Zentralteil von Rostrum und Barteln bildet ein Bindegewebe aus dicht gebündelten Fasern. Neben längsverlaufenden Bindegewebsbündeln, insbesondere im Zentrum, existieren radiär zur Peripherie ziehende Faserbündel sowie an der Grenze zum Bindegewebe hin konzentrische Fasergruppen (Abb. 14).

Unregelmäßig im Bindegewebe verteilt liegen vereinzelt kleine Gefäße und Kapillaren. Melanophoren sind beschränkt auf den Bindegewebsbereich dicht unter der Basalmembran. Ganz vereinzelt treten kleine Bündel von Dendriten in Erscheinung, die meist im peripheren Bindegewebe liegen.

Knorpelige Flemente, etwa als Derivate der Lippenknorpel, konnten weder im Rostrum noch in den Barteln festgestellt werden. Auch Muskulatur fehlt, die in Barteln von Welsen, jedenfalls an der Basis, durchaus auftreten kann.

Damit wären diese Kopfanhänge als bartelartige Hautprojektionen ohne besondere Stiitzelemente aufzufassen, die Sinnesorgane tragen.

\section{DISKUSSION}

Vergleicht man die Bauverhältnisse des Geruchsorgans von Rbinomuraena ambonensis mit denen anderer Knochenfische, so lassen sich am ehesten Ähnlichkeiten mit der Situation bei Anguilla anguilla konstatieren. Dies gilt besonders für die Gestalt und Ausdehnung der Riechhöhle, die Position ihrer Offnungen, als auch für Zahl, Größe und Anordnung der Riechfalten. Der bemerkenswerteste Unterschied liegt ohne Frage in der Existenz von Riechtrichtern bei Rbinomuraena ambonensis, die sich von den röhrenförmigen vorderen Riechöffnungen der Anguilliformes ableiten lassen.

Die Basis der Trichterröhre mündet direkt in den Raum, der zwischen den Innenrändern der Riechfalten gelegen ist und durch die schlitzförmige hintere Offnung ausmündet. Ohne Zweifel steht die Ausbildung eines solchen kohaerenten Systems: Trichteröffnung, Trichtermündung, zylindrischer Hohlraum zwischen den inneren Faltenrändern, hintere Offnung der Riechhöhle, im Dienste einer effektiven Duftwasserzirkulation, die aber erst dann gewährleistet ist, wenn trotz der Turbulenzen, die zwischen den Riechlamellen entstehen, ein Stau in der Riechhöhle selbst vermieden wird, so daß eine kontinuierliche Hauptdurchströmung gesichert ist. Daß dabei den Trichterorganen für die verschiedenen Bewegungsphasen eine zentrale Bedeutung zukommt, haben die Tuscheexperimente eindeutig erwiesen. In beiden erwähnten Fällen ist der Ventilationseffekt groß und die permanente Durchströmung mit Duftwasser gesichert. Dazu muß noch auf die hohe Durchströmgeschwindigkeit hingewiesen werden, die sich nach dem Bernoullischen Gesetz ergibt; denn das Verhältnis von Trichterquerschnitt (an der weitesten Stelle) zu Austrittsquerschnitt (Abb. 6) bedingt eine wesentliche Geschwindigkeitszunahme zur Austrittsstelle hin.

Die oben erwähnten Turbulenzen scheinen von nicht unbedeutender Rolle zu sein für die interlamelläre Mikroventilation und -zirkulation. Unter anderem ist davon abhängig die Heranführung der Duftstoffmoleküle an die im Schleim eingebetteten Riechhärchen. Bei einer gegebenen Menge von Duftmolekülen ist verständlicherweise die Anzahl der auf die Rezeptormembranen auftreffenden Molekiile um so größer, je stärker und zahlreicher die Turbulenzen sind, die in unmittelbarer Nähe der Riech- 
schleimhaut erzeugt werden. Damit läßt sich die Feststellung treffen, daß mit einer großen Riechfaltenzahl nicht nur eine große Riechfeldausdehnung verbunden ist, sondern daß auch die Häufigkeit der Mikroturbulenzen steigt.

Hinzu kommen die Strömungseffekte, die durch den Schlag der langen und dicht stehenden Flimmerhärchen ausgelöst werden, und die in der gleichen Weise wirksam sind. An keiner Stelle des Riechepithels fehlen diese Flimmerhärchen. Besonders dicht sind die Flimmerzellen im Epithel des inneren Randes der Riechfalten angeordnet.

Die Funktion dieser beweglichen Flimmerhärchen (Kinocilien) besteht weiterhin darin, die das Epithel überziehende Schleimschicht in permanenter Bewegung zu halten, was ohne Frage das Diffundieren von Duftmolekülen in den Schleim beschleunigt und damit ihren Kontakt mit der Rezeptormembran erheblich forciert (Holl 1965).

Betrachtet man alle morphologischen Bauprinzipien des Geruchsorgans von Rhinomuraena ambonensis in ihrer Gesamtheit und in der Abstimmung aufeinander, so läßt sich feststellen, daß zumindest von dieser Seite aus bestmögliche Voraussetzungen für eine hohe Leistungsfähigkeit des Gerudhsorgans existieren.

Wie die Lebendbeobachtung der im Seewasseraquarium gehälterten Rhinomuraena ambonensis zeigte, nehmen die beiden Trichter eine ganz bestimmte Position zur Körperlängsachse ein (siehe auch Abb. 2). Diese Ausrichtung ist unabhängig von der Aktivität des Tieres; sie ändert sich auch kaum, wenn der Fisch schnell vorwärts oder etwa rückwärts schwimmt (was relativ häufig der Fall ist). Damit erhebt sich aber die Frage nach den Voraussetzungen für die Form- und auch Haltungskonstanz der trichterförmigen Hautlappen, die selbst dann zu registrieren ist, wenn insbesondere die Trichterinnenfläche einem erhöhten Staudruck ausgesetzt ist.

Nach den lichtmikroskopischen Untersuchungen fehlen spezielle Stïtzelemente wie Knorpel oder Knochen, genauso wie durchaus denkbare schwellgewebeähnliche Strukturen. Um einer Beantwortung dieser Frage näherzukommen, wurden inzwischen elektronenmikroskopische Untersuchungen begonnen, die zugleich auch die Feinstruktur der intraepithelialen Nervenfaserbündel der Trichter zum Gegenstand haben.

Eine weitere Frage, die im Rahmen der vorliegenden Bearbeitung nur mit gewissem Vorbehalt zu diskutieren ist, aber von besonderer Wichtigkeit erscheint, erhebt sich, wenn auf die olfaktorische Orientierung von Rhinomuraena ambonensis eingegangen werden soll.

$\mathrm{Da}$ die beiden zur Verfügung stehenden lebenden Tiere für die licht- und elektronenmikroskopischen Untersuchungen bestimmt waren, konnten nur wenige, zeitlich sehr begrenzte und vom Methodischen her recht unzureichende Experimente ausgefuhrt werden. Dabei stellte sich heraus, daß beide Tiere auch nach mehr als achttägigem Hungern auf Körperextrakte von frischen Süßwasser- oder Seewasserfischen, tiefgefrorenen Garnelen und frischem Säugerfleisch nicht reagierten. Dieser Befund zeigt jedoch nur, daß für exakte Dressurversuche eine längere und sorgfältige Eingewöhnung der Tiere erforderlich ist. Außerdem liegt die Vermutung nahe, daß Rbinomuraena ambonensis ein Nahrungsspezialist ist, worauf auch die Beobachtungen von KuAUsEWITZ (1970) hindeuten.

Obwohl Rhinomuraena ambonensis ein morphologisch extrem ausgebildetes Geruchsorgan hat, scheint sie sich nicht unbedingt in gleichem Maße olfaktorisch zu orientieren wie Anguilla anguilla. Neben dem ohne Zweifel strömungstechnisch, aber 
auch hinsichtlich seiner Riechfeldausdehnung hervorragenden Geruchsorgan dürfte das Auge nicht ohne Bedeutung für die Orientierung sein. Trotzdem wäre es vertretbar, Rbinomuraena ambonensis in die Gruppe der makrosmatischen Knochenfische, deren bekanntester Vertreter der Aal ist, einzustufen, zumal die Bauverhältnisse des Geruchsorgans ganz eindeutig enge Beziehungen zu Anguilla anguilla aufzeigen lassen. Eine endgültige Beurteilung der Leistung des Geruchsorgans von Rhinomuraena ambonensis muß allerdings der Lebendbeobachtung im Biotop und Dressurexperimenten vorbehalten bleiben.

Teilweise ungeklärt blieb auch die Frage nach der Art der im Epithel der Trichter, des Rostrum und der Barteln nachgewiesenen Sinnesknospen, die stark den Geschmacksknospen der Knochenfische ähneln. Da bei Knochenfischen Geschmacksknospen und überhaupt chemorezeptorische Organe nicht allein auf die Mundhöhle beschränkt sind (BARDACH et al. 1967 u. a.), häufig dagegen gerade auf Barteln sitzen, liegt die Vermutung nahe, daß es sich bei diesen Sinnesknospen um chemische Sinnesorgane handeln könnte. Möglicherweise haben sie die Aufgabe, die Nahrung auf ihre Genießbarkeit zu prüfen.

Die Interpretation der reichen Innervierung des Trichterepithels von Rbinomuraena ambonensis kann erst nach Abschluß der begonnenen elektronenmikroskopischen Bearbeitung erfolgen.

\section{ZUSAMMENFASSUNG}

1. An drei, wahrscheinlich adulten Exemplaren der Nasenmuräne, Rbinomuraena ambonensis BARBOUR, wurden die funktionelle Morphologie des Geruchsorgans sowie dessen Histologie und die der bartelartigen Kopfanhänge untersucht.

2. Die Ränder der vorderen Riechöffnungen sind zu trichterförmigen Hautlappen ausgewachsen. Die in ihrer Ausrichtung und Form durch Körperbewegungen kaum beeinflußbaren Trichter leiten auch im Ruhezustand des Tieres Wasser in die Riechhöhle, da ihre weite Offnung im Strömungsbereich des Atemwassers liegt. Auf diese Weise erfolgt eine effektive Ventilation der Riechhöhle.

3. Im mehrschichtigen Epithel der Trichter liegen Sekretzellen und vereinzelt Sinnesknospen; unmittelbar über den Epithelbildungszellen verlaufen Nervenfasern. Im Bindegewebe, das beidseitig von Epithel bedeckt ist, liegen Gefäße, Nervenbündel (Nervus trigeminus) und Pigmentzellen. Muskelgewebe fehlt. Neben dem Bindegewebe gibt es kein anderes Stïtzgewebe.

4. Die beiden Riechhöhlen sind von extremer Ausdehnung. Die Zahl der lamellenartigen Riechfalten, die in zwei Doppelreihen nach dem 900-Typ liegen, beträgt ca. 200. Zwischen den Innenrändern der Falten einer Doppelreihe, dorsal von der Mittelraphe, erstreckt sich eine zylindrische Rinne, die der Duftwasserpassage dient.

5. Die Riechfalten sind bis auf die lateralen, dorsalen Teile und Teile der ventralen Faltenansätze kontinuierlich mit Riechepithel bedeckt. Der histologische Aufbau des Riechepithels entspricht dem des Aals. Die Rezeptorendichte schwankt zwischen $2-3 \times 10^{4}$ pro $\mathrm{mm}^{2}$. Im Verhältnis zur Riechhöhlenausdehnung ist die Riechfeldgröße erheblich. 
6. Tuscheexperimente zeigten, daß - ausgelöst durch die in die Längsrinne der Riechhöhle ragenden fingerförmigen Innenränder der Riechfalten - Mikroturbulenzen entstehen, die neben der Flimmerbewegung durch Kinocilien für die Mikroventilation des Faltensystems sorgen.

7. Das auf der Symphyse des Oberkiefers zwischen den Trichterbasen sitzende Rostrum ist eine Hautprojektion, deren bindegewebiger Zentralteil ein mehrschichtiges Epithel trägt. Im Bindegewebe sind Gefäße, feine Nerven und Pigmentzellen eingebettet. Im Epithel finden sich geschmacksknospenähnliche Sinnesorgane. Prinzipiell haben die drei Mandibularbarteln den gleichen histologischen Aufbau wie das Rostrum. Auch ihr Epithel weist Sinnesknospen auf, die wie jene des Rostrums und der Trichter vielleicht als chemische Sinnesorgane beim Nahrungstest fungieren.

8. Nach dem Ergebnis vorliegender Untersuchungen wird Rbinomuraena ambonensis in die Gruppe der makrosmatischen Knochenfische eingereiht.

Danksagung. Der Deutschen Forschungsgemeinschaft sei für Unterstützung bei der Durchführung dieser Untersuchungen gedankt.

\section{ZITIERTE LITERATUR}

BARDACH, J. E., FujYYA, M. \& Holl, A., 1967. Investigations of external chemoreceptors of fishes. Int. Symp. Olfac. Taste 2 (Tokyo), 647-665.

Burne, R. H., 1909. The anatomy of the olfactory organ of teleostean fishes. Proc. zool. Soc. Lond. 1909, 610-663.

HoLl, A., 1965. Vergleichende morphologische und histologische Untersuchungen am Gerudısorgan der Knochenfische. Z. Morph. Ökol. Tiere 54, 707-782.

- \& Mfrnel, W., 1969. Das Geruchsorgan des Tiefseefisches Aphanopus carbo (Percomorphi, Trichiuridae). Helgoländer wiss. Meeresunters. 18, 404-423.

Krausewitz, W., 1970. Die Nasenmuränen - zwei bemerkenswerte Neuimporte. AquarienMag. 4, 156-159.

KoBAyashI, K., 1962. A record of the muraenoid fish Rhinomuraena ambonensis (BARBouR) from Amami-Oshima. Bull. Fac. Fish. Hokkaido Univ. 13, 36-37,

Latbach, E., 1937. Das Geruchsorgan des Aals in seinen verschiedenen Entwicklungsstadien. Zool. Jb. (Abt. Anat. Ontog. Tiere) 63, 37-72.

PFEIfFer, W., 1964. The morphology of the olfactory organ of Hoplopagrus guentheri Gint 1862. Can. J. Zool. 42, 235-237.

Schultz, L. P., Herald, E. S., Lachner, E. A., Welander, A. D., \& Woods, L. P., 1953. Fishes of the Marshall and Marianas Islands. Vol. 1. Bull. U.S. natn. Mus. 202, 1-685.

Smith, J. L. B., 1962. The moray eels of the Red Sea and Western Indian Ocean. Ichthyol. Bull. 23, 421-444.

Teichmann, H., 1954. Vergleichende Untersuchungen an der Nase der Fische. Z. Morph. Okol. Tiere 43, 171-212.

WEBER, M. \& BEAUfORT, L. F. DE, 1916. The fishes of the Indo-Australian archipelago. III. Ostariophysi: II Cyprinoidea, Apodes, Synbranchi. E. J. Brill, Leiden, 455 pp.

Anschrift des erstgenannten Autors: Dr. A. Holl

I. Zoologisches Institut

der Universität Gießen

63 Gießen

Stephanstraße 24

Deutschland (BRD) 